\title{
Distributed Synthesis and Stability of Cooperative Distributed Model Predictive Control for Linear Systems *
}

\author{
Christian Conte $^{\mathrm{a}}$, Colin N. Jones ${ }^{\mathrm{b}}$, Manfred Morari ${ }^{\mathrm{a}}$, Melanie N. Zeilinger ${ }^{\mathrm{c}}$ \\ ${ }^{a}$ Automatic Control Laboratory, Department of Information Technology and Electrical Engineering, ETH Zurich, 8092 Zurich, Switzerland \\ ${ }^{\mathrm{b}}$ Automatic Control Laboratory, École Polytechnique Fédérale de Lausanne (EPFL), 1015 Lausanne, Switzerland
}

${ }^{\mathrm{c}}$ Institute for Dynamic Systems and Control, Department of Mechanical and Process Engineering, ETH Zurich, 8092 Zurich, Switzerland

\begin{abstract}
This paper presents a new formulation and synthesis approach for stabilizing cooperative distributed model predictive control (MPC) for networks of linear systems, which are coupled in their dynamics. The controller is defined by a network-wide constrained optimal control problem, which is solved online by distributed optimization. The main challenge is the definition of a global MPC problem, which both defines a stabilizing control law and is amenable to distributed optimization, i.e., can be split into a number of appropriately coupled subproblems. For such a combination of stability and structure, we propose the use of a separable terminal cost function, combined with novel time-varying local terminal sets. For synthesis, we introduce a method that allows for constructing these components in a completely distributed way, without central coordination. The paper covers the nominal case in detail and discusses the extension of the methodology to reference tracking. Closed-loop functionality of the controller is illustrated by a numerical example, which highlights the effectiveness of the proposed controller and its time-varying local terminal sets.
\end{abstract}

Key words: Distributed control; predictive control; large-scale systems.

\section{Introduction}

Control of large-scale networks of dynamic systems is a challenging problem, in particular if the systems in the network are subject to communication constraints as well as constraints on states and inputs. MPC is a well-established methodology for the control of constrained systems. Its application under communication constraints has been a field of active research in recent years, with applications in fields such as power networks (Venkat, Hiskens, Rawlings \& Wright 2008) and building automation (Ma, Richter \& Borrelli 2012). Two key challenges in distributed MPC are closed-loop stability and controller synthesis under distributed computations. This paper addresses these challenges and proposes a less restrictive solution approach compared to methods currently available in the literature.

In order to obtain a distributed MPC formulation with sta-

\footnotetext{
* This paper was not presented at any IFAC meeting. Corresponding author C. Conte. Tel. +41-78-6522388.

Email addresses: christian.contedsiemens.com (Christian Conte), colin.jones@epfl.ch (Colin N. Jones), morari@control.ee.ethz.ch (Manfred Morari), mzeilinger@ethz.ch (Melanie N. Zeilinger).
}

bility guarantees, results from unconstrained decentralized and distributed control can be used. In decentralized control, the controllers in the network do not exchange information, while in distributed control they do. Important findings related to the analysis of decentralized systems are summarized in (Šiljak 1991), where especially vector Lyapunov functions are used for stability analysis. Synthesis approaches for distributed control laws based on linear matrix inequalities have been proposed, e.g., in (Langbort, Chandra \& D'Andrea 2004) and (Zečević \& Šiljak 2010).

The literature on distributed MPC mainly distinguishes between non-cooperative and cooperative approaches. In non-cooperative distributed MPC, e.g. (Farina \& Scattolini 2012), neighboring systems typically communicate once per time-step and each system is equipped with a local MPC controller that acts selfishly and is robust against coupling to neighboring systems. While requiring less communication, non-cooperative approaches can become very conservative or even infeasible in presence of strong dynamic coupling. In cooperative distributed MPC, as e.g. in (Venkat, Rawlings \& Wright 2005) or more recently (Giselsson \& Rantzer 2013), neighboring systems typically communicate several times per time-step in order to solve a globally defined MPC problem by distributed optimization. 
A key requirement is for the MPC problem to be structured such that distributed optimization methods are applicable.

While some cooperative distributed MPC approaches derive stability guarantees based on long horizons (Giselsson \& Rantzer 2013), most approaches rely on terminal costs and terminal invariant sets (Mayne, Rawlings, Rao \& Scokaert 2000). However, standard terminal costs and invariant sets are based on global Lyapunov stability and invariance concepts and do not exhibit a structure which is amenable to distributed optimization. In order to obtain such a structure for the terminal cost, vector Lyapunov functions (Šiljak 1991) or linear matrix inequality (LMI) based methods (Langbort et al. 2004), (Zečević \& Šiljak 2010) can be used. As for a structured terminal cost, however, the available methods are limited. One possibility is the use of a trivial terminal set, i.e. a point, as suggested in (Stewart, Venkat, Rawlings, Wright \& Pannocchia 2010), which will, however, reduce the size of the region of attraction of the resulting MPC controller. Another option is to resort to robust positively invariant sets (Maestre, Muñoz de la Peña, Camacho \& Alamo 2011), considering dynamic coupling as a disturbance. In presence of strong coupling however, the resulting terminal sets tend to be small or may even be empty. Another possibility is the use of time-varying local sets, as suggested in (Raković, Kern \& Findeisen 2010), the synthesis of which is however non-obvious and not discussed in the paper.

The contribution of this paper is twofold and builds on (Conte, Voellmy, Zeilinger, Morari \& Jones 2012). The first contribution, based on (Jokić \& Lazar 2009), is a novel concept for time-varying local terminal sets leading to a cooperative distributed MPC controller with closed-loop stability guarantee. The proposed methodology can be used to construct terminal sets for both regulation and reference tracking MPC. As opposed to the concept presented in (Raković et al. 2010), the set dynamics advocated in this paper are directly linked to the system dynamics and stability properties. The second contribution is a practical distributed synthesis method for networks of linear systems with quadratic costs and polytopic constraints. This method can be executed in a completely distributed way, a feature which is particularly beneficial in case of changing network topologies, where new controllers have to be synthesized on the fly without central coordination. This case has received considerable attention in the context of plug-and-play MPC (Riverso, Farina \& Trecate 2013), (Zeilinger, Pu, Riverso, Ferrari-Trecate \& Jones 2013).

In Section 2, preliminaries on distributed systems and MPC are introduced. In Section 3, the formulation of the nominal cooperative distributed MPC controller is presented and in Section 4 , its distributed synthesis is discussed. Section 5 summarizes the nominal case and Section 6 covers the extension to reference tracking. In Section 7, a numerical example is provided and Section 8 concludes the paper.

\section{Preliminaries}

\subsection{Notation}

The set $\{1, \ldots, M\} \subseteq \mathbb{N}$ is denoted as $\mathcal{M}$. A block-diagonal matrix $S$ with blocks $S_{i}$, where $i \in \mathcal{M}$, is denoted as $S=$ $\operatorname{diag}_{i \in \mathcal{I}}\left(S_{i}\right)$ or $S=\operatorname{diag}\left(S_{1}, \ldots, S_{M}\right)$, depending on the context. Similarly, a vector which consists of the stacked subvectors $x_{i}, i \in \mathcal{I} \subseteq \mathbb{N}$, is denoted as $\operatorname{col}_{i \in \mathcal{I}}\left(x_{i}\right)$ or $\operatorname{col}\left(x_{1}, \ldots, x_{M}\right)$. If a matrix $S$ is positive definite, we write $S>0$ and if it is positive semi-definite, we write $S \geq 0$. The $n$-dimensional identity matrix is denoted as $I_{n}$. A function $\beta(\cdot): \mathbb{R}_{+} \rightarrow \mathbb{R}_{+}$is of class $\mathcal{K}$ if it is continuous, strictly increasing and if $\beta(0)=0$. It is of class $\mathcal{K}_{\infty}$ if additionally it holds that $\lim _{s \rightarrow \infty} \beta(s)=\infty$.

\subsection{Distributed Linear Time-Invariant (LTI) Systems}

We consider a network of $M$ linear time-invariant systems, where each system $i \in \mathcal{M}$ has a state $x_{i} \in \mathbb{R}^{n_{i}}$, an input $u_{i} \in \mathbb{R}^{m_{i}}$ and an output $y_{i} \in \mathbb{R}^{p_{i}}$. We consider systems that are coupled in the state and in the output. The dynamics of the local systems can thus be written as

$$
x_{i}^{+}=\sum_{j=1}^{M} A_{i j} x_{j}+B_{i} u_{i}, \quad y_{i}=\sum_{j=1}^{M} C_{i j} x_{j} \quad \forall i \in \mathcal{M}
$$

where $A_{i j} \in \mathbb{R}^{n_{i} \times n_{j}}, B_{i} \in \mathbb{R}^{n_{i} \times m_{i}}$ and $C_{i j} \in \mathbb{R}^{p_{i} \times n_{j}}$. Note that additional coupling in the inputs could be easily reformulated into the form in (1) by defining the original inputs as additional states and the changes in the original inputs as the new inputs. The coupling in states and outputs is used to define the notion of neighboring systems.

Definition 1 (Neighboring Systems) System $j$ is a neighbor of system $i$ if $A_{i j} \neq 0$ or $C_{i j} \neq 0$. The set of all neighbors of $i$, including $i$ itself, is denoted as $\mathcal{N}_{i}$. The states of all systems $j \in \mathcal{N}_{i}$ are denoted as $x_{\mathcal{N}_{i}}=\operatorname{col}_{j \in \mathcal{N}_{i}}\left(x_{j}\right) \in \mathbb{R}^{n_{\mathcal{N}_{i}}}$.

The local systems (1) can thus, with matrices of appropriate dimensions, be equivalently written as

$$
x_{i}^{+}=A_{\mathcal{N}_{i}} x_{\mathcal{N}_{i}}+B_{i} u_{i}, \quad y_{i}=C_{\mathcal{N}_{i}} x_{\mathcal{N}_{i}} \quad \forall i \in \mathcal{M} .
$$

Throughout the paper, it is assumed that neighboring systems can communicate with each other.

Assumption 2 (Communication) Two systems $i$ and $j$ can communicate, in a bidirectional way, if $i \in \mathcal{N}_{j}$ or $j \in \mathcal{N}_{i}$.

Both the local states and inputs are subject to constraints

$$
x_{i} \in \mathcal{X}_{i}, \quad u_{i} \in \mathcal{U}_{i} \quad \forall i \in \mathcal{M},
$$

where for each $i \in \mathcal{M}, \mathcal{X}_{i} \subseteq \mathbb{R}^{n_{i}}$ and $\mathcal{U}_{i} \subseteq \mathbb{R}^{m_{i}}$ are convex sets which contain the origin in their interior. 
By combining the local system dynamics in (1), the linear dynamics of the global system result in

$$
x^{+}=A x+B u, y=C x,
$$

where $x=\operatorname{col}_{i \in \mathcal{M}}\left(x_{i}\right) \in \mathbb{R}^{n}, u=\operatorname{col}_{i \in \mathcal{M}}\left(u_{i}\right) \in \mathbb{R}^{m}$ and $y=\operatorname{col}_{i \in \mathcal{M}}\left(y_{i}\right) \in \mathbb{R}^{p}$. At some points in the paper, the equivalent notation $x^{t+1}=A x^{t}+B u^{t}$ will be used to emphasize the current time index. The global system matrix $A \in \mathbb{R}^{n \times n}$ and the global output map $C \in$ $\mathbb{R}^{p \times n}$ are block-sparse with entries $A_{i j}$ and $C_{i j}$ for each $(i, j) \in \mathcal{M}^{2}$, provided $j \in \mathcal{N}_{i}$, and the global input map $B=\operatorname{diag}_{i \in \mathcal{M}}\left(B_{i}\right) \in \mathbb{R}^{n \times m}$ is block-diagonal. Similarly, combining the local state and input constraints (3), the global constraints for system (4) result in

$x \in \mathcal{X}:=\mathcal{X}_{1} \times . . \times \mathcal{X}_{M} \subseteq \mathbb{R}^{n}, u \in \mathcal{U}:=\mathcal{U}_{1} \times . . \times \mathcal{U}_{M} \subseteq \mathbb{R}^{m}$

In order for the methodology in this paper to apply, we make the following assumption on the stabilizability of $(A, B)$.

Assumption 3 (Structured Linear Controller) There exists a linear control law of the form

$$
\kappa_{\mathrm{f}}(x):=K_{\mathrm{f}} x=\operatorname{col}_{i \in \mathcal{M}}\left(K_{\mathcal{N}_{i}} x_{\mathcal{N}_{i}}\right)
$$

where $K_{\mathrm{f}} \in \mathbb{R}^{m \times n}$ and $K_{\mathcal{N}_{i}} \in \mathbb{R}^{m_{i} \times n_{\mathcal{N}_{i}}} \forall i \in \mathcal{M}$, such that the system $x^{+}=A x+B \kappa_{\mathrm{f}}(x)$ is asymptotically stable.

Given a stabilizing controller $\kappa_{\mathrm{f}}(x)$, the notion of a positively invariant set can be defined.

Definition 4 (Positively Invariant (PI) Set) $A$ set $\mathcal{X}_{\mathrm{f}}$ is PI for the dynamics $x^{+}=A x+B \kappa_{\mathrm{f}}(x)$, if $x \in \mathcal{X}_{\mathrm{f}} \Rightarrow x^{+} \in \mathcal{X}_{\mathrm{f}}$.

It is important to note that even if $\kappa_{\mathrm{f}}(x)$ is structured as in (6), any PI set according to Definition 4 is globally defined and does not generally exhibit structure. In particular, it is generally not a Cartesian product of local sets.

\subsection{Nominal Centralized MPC}

The nominal MPC control law for regulation is defined through the finite horizon optimal control problem

$$
\begin{aligned}
& V^{*}(x)=\min _{\mathbf{x}, \mathbf{u}} V_{\mathbf{f}}(x(N))+\sum_{k=0}^{N-1} l(x(k), u(k)) \\
\text { s.t. } & x(0)=x, \\
& x(k+1)=A x(k)+B u(k) \forall k \in\{0, . ., N-1\}, \\
& (x(k), u(k)) \in \mathcal{X} \times \mathcal{U} \forall k \in\{0, . ., N-1\}, \\
& x(N) \in \mathcal{X}_{\mathrm{f}}
\end{aligned}
$$

which is in the following referred to as the nominal MPC problem. We define $\mathcal{X}_{N} \subseteq \mathbb{R}^{n}$ as the set of initial states $x$, for which (7) is feasible. Both the stage cost $l(x, u)$ and the terminal cost $V_{\mathrm{f}}(x)$ are positive definite convex functions and the terminal set $\mathcal{X}_{\mathrm{f}} \subseteq \mathbb{R}^{n}$ is convex, compact and contains the origin in the interior. Furthermore, $\mathbf{u}=$ $\{u(0), \ldots, u(N-1)\}$ denotes an input sequence over the finite horizon $N$ and $\mathbf{u}^{*}(x)=\left\{u^{*}(x, 0), \ldots, u^{*}(x, N-1)\right\}$ denotes the sequence minimizing (7) for the initial state $x$. The first element of $\mathbf{u}^{*}(x)$ defines the state feedback control law $\kappa_{\mathrm{MPC}}(x):=u^{*}(x, 0)$. Sufficient conditions for asymptotic stability under $\kappa_{\mathrm{MPC}}(x)$ are given in the following.

Theorem 5 ((Mayne et al. 2000)) Let $\beta_{1}(\cdot)$ and $\beta_{2}(\cdot)$ be $\mathcal{K}_{\infty}$ class functions. If $\mathcal{X}_{\mathrm{f}} \subseteq \mathcal{X}$ and (8) holds $\forall x \in \mathcal{X}_{\mathrm{f}}$, then the closed-loop system $x^{+}=A x+B \kappa_{\mathrm{MPC}}(x)$ is asymptotically stable on the domain $\mathcal{X}_{N}$ :

$$
\begin{aligned}
& \kappa_{\mathrm{f}}(x) \in \mathcal{U}, A x+B \kappa_{\mathrm{f}}(x) \in \mathcal{X}_{\mathrm{f}}, \\
& \beta_{1}\left(\|x\|_{2}\right) \leq V_{\mathrm{f}}(x) \leq \beta_{2}\left(\|x\|_{2}\right) \\
& V_{\mathrm{f}}\left(A x+B \kappa_{\mathrm{f}}(x)\right)-V_{\mathrm{f}}(x) \leq-l\left(x, \kappa_{\mathrm{f}}(x)\right)
\end{aligned}
$$

By $(8 \mathrm{a}), \mathcal{X}_{\mathrm{f}}$ is required to be a feasible PI set and by (8b) and $(8 \mathrm{c}), V_{\mathrm{f}}(x)$ is required to be a Lyapunov function for $x^{+}=A x+B \kappa_{\mathrm{f}}(x)$ on the domain $\mathcal{X}_{\mathrm{f}}$.

\subsection{Synthesis of Nominal Centralized MPC Controllers for LTI Systems and Polytopic Constraints}

The main difficulty in the synthesis of stabilizing nominal MPC controllers is the construction of a terminal cost $V_{\mathrm{f}}(x)$ and a terminal set $\mathcal{X}_{\mathrm{f}}$ satisfying (8). Synthesis methods for these components are outlined in the following and will form the basis for the distributed synthesis techniques in Section 4.

\subsubsection{Centralized Terminal Cost Synthesis}

For linear systems, the terminal cost is typically chosen to be quadratic, i.e. $V_{\mathrm{f}}(x)=x^{T} P_{\mathrm{f}} x$, and the terminal controller to be linear, i.e. $\kappa_{\mathrm{f}}(x)=K_{\mathrm{f}} x$. Given a quadratic stage cost $l(x, u)=x^{T} Q x+u^{T} R u$, with $Q \geq 0$ and $R>0$, conditions $(8 \mathrm{~b})$ and $(8 \mathrm{c})$ can be reformulated as an LMI in $\left(P_{\mathrm{f}}, K_{\mathrm{f}}\right)$, i.e.

$$
\left[\begin{array}{cccc}
E & E A^{T}+Y^{T} B^{T} & E Q^{\frac{1}{2}} & Y^{T} R^{\frac{1}{2}} \\
A E+B Y & E & 0 & 0 \\
Q^{\frac{1}{2}} E & 0 & I & 0 \\
R^{\frac{1}{2}} Y & 0 & 0 & I
\end{array}\right] \geq 0
$$

where $E:=P_{\mathrm{f}}^{-1}$ and $Y:=K_{\mathrm{f}} E$, for details see (Boyd, Ghaoui, Feron \& Balakrishnan 1994). Note that (9) is obtained from the Lyapunov decrease condition (8c) by applying Schur complement techniques. In combination with a suitable objective function, e.g. minimization of $-\log \operatorname{det} E$ for maximizing the volume of the 1-level set ellipsoid $\mathcal{E}:=$ $\left\{x \in \mathbb{R}^{n} \mid x^{T} P_{\mathrm{f}} x \leq 1\right\}, P_{\mathrm{f}}$ can be computed by efficient numerical tools for semi-definite programming. 


\subsubsection{Centralized Terminal Set Synthesis}

In the following, a design method for ellipsoidal PI terminal sets is given. Ellipsoidal PI sets are superior compared to polytopic sets in terms of scalability of the synthesis methods. Consider state and input constraints $\mathcal{X}=\{x \in$ $\left.\mathbb{R}^{n} \mid H_{x} x \leq h_{x}\right\}$ and $\mathcal{U}=\left\{u \in \mathbb{R}^{m} \mid H_{u} u \leq h_{u}\right\}$, with $H_{x} \in \mathbb{R}^{l_{x} \times \bar{n}}, h_{x} \in \mathbb{R}^{l_{x}}, H_{u} \in \mathbb{R}^{l_{u} \times m}, h_{u} \in \mathbb{R}^{\bar{l}_{u}}$. Consider furthermore a linear terminal control law $\kappa_{\mathrm{f}}(x)=K_{\mathrm{f}} x$ and a quadratic terminal cost $V_{\mathrm{f}}(x)=x^{T} P_{\mathrm{f}} x$. If the terminal cost fulfills (8), it is a Lyapunov function and its level sets $\mathcal{X}_{\mathrm{f}}=\left\{x \in \mathbb{R}^{n} \mid x^{T} P_{\mathrm{f}} x \leq \alpha\right\}$ are PI for the system dynamics $x^{+}=\left(A+B K_{\mathrm{f}}\right) x$ (Blanchini 1999). The problem of finding the largest feasible level set of $V_{\mathrm{f}}(x)$ can be posed as

$$
\begin{aligned}
\max _{\alpha} \alpha & \\
\text { s.t. } & \sigma_{\mathcal{E}}\left(H_{x, i}^{T}\right)^{2} \alpha \leq h_{x, i}^{2} \forall i \in\left\{1, \ldots, l_{x}\right\} \\
& \sigma_{\mathcal{E}}\left(K_{\mathrm{f}}^{T} H_{u, j}^{T}\right)^{2} \alpha \leq h_{u, j}^{2} \forall j \in\left\{1, \ldots, l_{u}\right\}
\end{aligned}
$$

where $\left(H_{x, i}, h_{x, i}\right)$ and $\left(H_{u, j}, h_{u, j}\right)$ correspond to the $i$ th and $j$ th halfspace constraint of the polytopes $\mathcal{X}$ and $\mathcal{U}$ respectively. Furthermore, the function $\sigma_{\mathcal{E}}(a):=\max _{x \in \mathcal{E}} a^{T} x=$ $\left\|P_{\mathrm{f}}^{-\frac{1}{2}} a\right\|_{2}$, where $a \in \mathbb{R}^{n}$, is the support function of the 1 level set $\mathcal{E}$ defined above and is available in explicit form since $\mathcal{E}$ is ellipsoidal. Note that the equivalence $a^{T} x \leq$ $b \forall x \in \mathcal{E} \Leftrightarrow \sigma_{\mathcal{E}}(a) \leq b$ holds. Problem (10) is a linear program (LP) in only one variable, for which a variety of efficient solution methods are available.

\section{Stability of Nominal Cooperative Distributed MPC}

\subsection{Problem Statement}

In cooperative distributed MPC, the global MPC problem (7) is solved online by distributed optimization. A variety of distributed optimization algorithms are available in the literature, e.g. the dual decomposition based subgradient method or the alternating direction method of multipliers (ADMM), see (Bertsekas \& Tsitsiklis 1989). Most distributed optimization algorithms work conceptually similar:

- The network-wide optimization problem is decomposed into a number of subproblems, each of which is assigned to one system in the network.

- In consecutive rounds, the subproblems are solved, local solutions are shared among neighbors, and local firstorder steps towards the global optimizer are taken.

In presence of communication constraints according to Assumption 2, the key requirement for the application of distributed optimization is thus, that (7) is decomposable into $M$ subproblems, each of which involves variables $\left(x_{\mathcal{N}_{i}}, u_{i}\right)$ only. Inspecting problem (7), we recognize that the constraints (7b)-(7d) fulfill this property, and the stage cost $l(x, u)$ can be chosen separable, i.e. as a sum of terms
$l_{i}\left(x_{\mathcal{N}_{i}}, u_{i}\right)$. The main challenge is the construction of a separable terminal cost $V_{\mathrm{f}}(x)$ and a structured terminal set $\mathcal{X}_{\mathrm{f}}$, fulfilling (8) for stability. In this paper, we impose the particular structure

$$
\begin{aligned}
V_{\mathrm{f}}(x) & =\sum_{i=1}^{M} V_{\mathrm{f}, i}\left(x_{i}\right) \\
\mathcal{X}_{\mathrm{f}}\left(\alpha_{i}, \ldots, \alpha_{M}\right) & =\mathcal{X}_{\mathrm{f}, 1}\left(\alpha_{1}\right) \times \ldots \times \mathcal{X}_{\mathrm{f}, M}\left(\alpha_{M}\right),
\end{aligned}
$$

where $\mathcal{X}_{\mathrm{f}}\left(\alpha_{i}, \ldots, \alpha_{M}\right) \subseteq \mathbb{R}^{n}$ is a Cartesian product of parametrized local sets $\mathcal{X}_{\mathrm{f}}\left(\alpha_{i}\right)$. In Section 3.2 we show how a separable terminal cost (11a) can be formulated and in Section 3.3 we show how a terminal set $(11 \mathrm{~b})$ can be defined.

\subsection{Separable Terminal Cost}

A simple approach to construct a terminal cost function of form (11a), fulfilling (8b) and (8c), would be to require each function $V_{\mathrm{f}, i}\left(x_{i}\right)$ to decrease at every time step under a given terminal control law. Such an approach would, however, be very conservative. Consider for instance a system $i$ whose state $x_{i}$ rests in the origin, where $V_{\mathrm{f}, i}\left(x_{i}\right)=0$. If the state $x_{j}$ of a neighboring subsystem $j \in \mathcal{N}_{i}$ is nonzero, $x_{i}$ will necessarily be driven out of the origin, causing $V_{\mathrm{f}, i}\left(x_{i}\right)$ to increase. Therefore, as proposed in (Jokić \& Lazar 2009), it is desirable to allow the local terminal cost to increase, as long as at the same time the global terminal cost decreases, which is formalized in the following theorem.

\section{Theorem 6 (Theorem III.4 in (Jokić \& Lazar 2009))}

Let there be a structured control law $\kappa_{\mathrm{f}}(x)$ according to Assumption 3. Let $\mathcal{X}_{\mathrm{f}} \subseteq \mathbb{R}^{n}$ be a PI set as defined in Definition 4. If there exist, $\forall i \in \mathcal{M}$, functions $V_{\mathrm{f}, i}\left(x_{i}\right), \gamma_{i}\left(x_{\mathcal{N}_{i}}\right)$ and $l_{i}\left(x_{\mathcal{N}_{i}}, \kappa_{\mathcal{N}_{i}}\left(x_{\mathcal{N}_{i}}\right)\right)$, as well as functions $\beta_{1, i}(\cdot), \beta_{2, i}(\cdot)$ and $\beta_{3, i}(\cdot) \in \mathcal{K}_{\infty}$, such that $\forall x=\operatorname{col}_{i \in \mathcal{M}}\left(x_{i}\right) \in \mathcal{X}_{\mathrm{f}}$ the following conditions (12) hold, then $V_{\mathrm{f}}(x)=\sum_{i=1}^{M} V_{\mathrm{f}, i}\left(x_{i}\right)$ is a Lyapunov function for the system $x^{+}=A x+B \kappa_{\mathrm{f}}(x)$ on $\mathcal{X}_{\mathrm{f}}$.

$$
\begin{gathered}
\beta_{1, i}\left(\left\|x_{i}\right\|\right) \leq V_{\mathrm{f}, i}\left(x_{i}\right) \leq \beta_{2, i}\left(\left\|x_{i}\right\|\right) \quad \forall i \in \mathcal{M}, \\
\beta_{3, i}\left(\left\|x_{\mathcal{N}_{i}}\right\|\right) \leq l_{i}\left(x_{\mathcal{N}_{i}}, \kappa_{\mathcal{N}_{i}}\left(x_{\mathcal{N}_{i}}\right)\right) \quad \forall i \in \mathcal{M}, \\
V_{\mathrm{f}, i}\left(x_{i}^{+}\right)-V_{\mathrm{f}, i}\left(x_{i}\right) \leq \\
-l_{i}\left(x_{\mathcal{N}_{i}}, \kappa_{\mathcal{N}_{i}}\left(x_{\mathcal{N}_{i}}\right)\right)+\gamma_{i}\left(x_{\mathcal{N}_{i}}\right) \quad \forall i \in \mathcal{M}, \\
\sum_{i=1}^{M} \gamma_{i}\left(x_{\mathcal{N}_{i}}\right) \leq 0 .
\end{gathered}
$$

Conditions (12c) and (12d) ensure that while single terms $V_{\mathrm{f}, i}\left(x_{i}\right)$ may increase in some time steps, where the increase is bounded by functions $\gamma_{i}\left(x_{\mathcal{N}_{i}}\right)$, the global function $V_{\mathrm{f}}(x)$ decreases in every time step. This property can be used to define time-varying local terminal sets, as shown in the next section. 


\subsection{Time-Varying Local Terminal Sets}

The main idea is to define local terminal sets as level sets of the local terminal cost functions, i.e. $\mathcal{X}_{\mathrm{f}, i}\left(\alpha_{i}\right)=\left\{x_{i} \in\right.$ $\left.\mathbb{R}^{n_{i}} \mid V_{\mathrm{f}, i}\left(x_{i}\right) \leq \alpha_{i}\right\}$, where $0 \leq \alpha_{i}, \forall i \in \mathcal{M}$. For static local values $\alpha_{i}$ such level sets are, however, not invariant. In particular, assuming (12), $x_{i} \in \mathcal{X}_{\mathrm{f}, i}\left(\alpha_{i}\right) \nRightarrow A_{\mathcal{N}_{i}} x_{\mathcal{N}_{i}}+$ $B_{i} \kappa_{\mathcal{N}_{i}}\left(x_{\mathcal{N}_{i}}\right) \in \mathcal{X}_{\mathrm{f}, i}\left(\alpha_{i}\right)$ since, as time evolves, the state of any system $i$ might leave $\mathcal{X}_{\mathrm{f}, i}\left(\alpha_{i}\right)$ due to an increase in $V_{\mathrm{f}, i}\left(x_{i}\right)$. The key idea for addressing this issue is to update the size of the local terminal sets in each time step, using the previously introduced relaxation functions $\gamma_{i}\left(x_{\mathcal{N}_{i}}\right)$.

Definition 7 (Local time-varying terminal sets) Let $\kappa_{\mathrm{f}}\left(x^{t}\right)$ be a terminal control law satisfying Assumption 3 and let there be a separable terminal cost $V_{\mathrm{f}}\left(x^{t}\right)=\sum_{i=1}^{M} V_{\mathrm{f}, i}\left(x_{i}^{t}\right)$, with corresponding relaxation functions $\gamma_{i}\left(x_{\mathcal{N}_{i}}\right) \forall i \in \mathcal{M}$, satisfying (12). Define $\alpha$ such that $\mathcal{X}_{\mathrm{f}}^{\text {glob }}:=\left\{x \in \mathbb{R}^{n} \mid V_{\mathrm{f}}(x) \leq \alpha\right\} \subseteq \mathcal{X}$ and $\forall x \in \mathcal{X}_{\mathrm{f}}^{\text {glob }}: \kappa_{\mathrm{f}}(x) \in \mathcal{U}$. Local terminal sets are defined as

$$
\mathcal{X}_{\mathrm{f}, i}\left(\alpha_{i}^{t}\right):=\left\{x_{i} \in \mathbb{R}^{n_{i}} \mid V_{\mathrm{f}, i}\left(x_{i}\right) \leq \alpha_{i}^{t}\right\} \quad \forall i \in \mathcal{M}
$$

where the sizes $\alpha_{i}^{t}$ are defined by the set dynamics

$$
\alpha_{i}^{t+1}=\alpha_{i}^{t}+\gamma_{i}\left(x_{\mathcal{N}_{i}}^{t}\right) \forall i \in \mathcal{M}
$$

with $\sum_{i=1}^{M} \alpha_{i}^{0} \leq \alpha$ and $0 \leq \alpha_{i}^{0} \forall i \in \mathcal{M}$. A global terminal set for the MPC problem (7) is then defined as

$$
\mathcal{X}_{\mathrm{f}}\left(\alpha_{1}^{t}, \ldots, \alpha_{M}^{t}\right):=\mathcal{X}_{\mathrm{f}, 1}\left(\alpha_{1}^{t}\right) \times \ldots \times \mathcal{X}_{\mathrm{f}, M}\left(\alpha_{M}^{t}\right)
$$

As the global terminal set (15) is a Cartesian product, it is amenable to distributed optimization, it does however not obviously fulfill the MPC stability conditions (8) due to its time-varying nature. Therefore, in the following, it is shown that Definition 7 provides sufficient conditions for (8), i.e., (i) the local system states remain within the local timevarying terminal sets under the terminal control law, and (ii) the Cartesian product of these local terminal sets(15) is recursively feasible. Property (i) is shown in the following.

Lemma 8 If there are sets $\mathcal{X}_{\mathrm{f}, i}\left(\alpha_{i}^{t}\right) \forall i \in \mathcal{M}$, as defined in Definition 7, then the following holds:

$$
\begin{aligned}
& x_{i}^{t} \in \mathcal{X}_{\mathrm{f}, i}\left(\alpha_{i}^{t}\right) \Rightarrow x_{i}^{t+1} \in \mathcal{X}_{\mathrm{f}, i}\left(\alpha_{i}^{t+1}\right) \forall i \in \mathcal{M} \\
& 0 \leq \alpha_{i}^{t+1} \forall i \in \mathcal{M},
\end{aligned}
$$

where $x_{i}^{t+1}=A_{\mathcal{N}_{i}} x_{\mathcal{N}_{i}}^{t}+B_{i} \kappa_{\mathcal{N}_{i}}\left(x_{\mathcal{N}_{i}}^{t}\right) \in \mathcal{X}_{\mathrm{f}, i}\left(\alpha_{i}^{t+1}\right)$.

Proof: By Definition 7, $V_{\mathrm{f}, i}\left(x_{i}\right)$ fulfills (12a) and is therefore positive definite. Thus, $x_{i}^{t} \in \mathcal{X}_{\mathrm{f}, i}\left(\alpha_{i}^{t}\right)$ implies $0 \leq V_{\mathrm{f}, i}\left(x_{i}^{t}\right) \leq$ $\alpha_{i}^{t}$. By Definition 7, there exist $V_{\mathrm{f}, i}\left(x_{i}\right), \gamma_{i}\left(x_{\mathcal{N}_{i}}\right), \kappa_{\mathcal{N}_{i}}\left(x_{\mathcal{N}_{i}}\right)$ and $l_{i}\left(x_{\mathcal{N}_{i}}, \kappa_{\mathcal{N}_{i}}\left(x_{\mathcal{N}_{i}}\right)\right)$, such that by (12c) it holds that

$$
\begin{aligned}
0 & \leq V_{\mathrm{f}, i}\left(A_{\mathcal{N}_{i}} x_{\mathcal{N}_{i}}^{t}+B_{i} \kappa_{\mathcal{N}_{i}}\left(x_{\mathcal{N}_{i}}^{t}\right)\right) \\
& \leq V_{\mathrm{f}, i}\left(x_{i}^{t}\right)-l_{i}\left(x_{\mathcal{N}_{i}}^{t}, \kappa_{\mathcal{N}_{i}}\left(x_{\mathcal{N}_{i}}^{t}\right)\right)+\gamma_{i}\left(x_{\mathcal{N}_{i}}^{t}\right) \\
& \leq V_{\mathrm{f}, i}\left(x_{i}^{t}\right)+\gamma_{i}\left(x_{\mathcal{N}_{i}}^{t}\right) \leq \alpha_{i}^{t}+\gamma_{i}\left(x_{\mathcal{N}_{i}}^{t}\right)=\alpha_{i}^{t+1}
\end{aligned}
$$

which proves properties (16).

Even if, as shown above in Lemma 8, the local system states remain within the time-varying local terminal sets (13), it is not obvious that the Cartesian product (15) of these sets is recursively feasible w.r.t. the global state and input constraints. It can, however, be shown that if the global terminal set in (15), at $t=0$, is a subset of $\mathcal{X}_{\mathrm{f}}^{\text {glob }}$, which, as a level set of the Lyapunov function $V_{\mathrm{f}}(x)$ is PI w.r.t. $x^{t+1}=A x^{t}+B \kappa_{\mathrm{f}}\left(x^{t}\right)$, then it remains in $\mathcal{X}_{\mathrm{f}}^{\text {glob }}$ at all future time steps. This property is shown in the following Lemma.

Lemma 9 If there exist sets $\mathcal{X}_{\mathrm{f}}^{\text {glob }}$ and $\mathcal{X}_{\mathrm{f}, i}\left(\alpha_{i}^{t}\right) \forall i \in \mathcal{M}$, as defined in Definition 7, then property (17) holds:

$$
\mathcal{X}_{\mathrm{f}, 1}\left(\alpha_{1}^{t}\right) \times \ldots \times \mathcal{X}_{\mathrm{f}, M}\left(\alpha_{M}^{t}\right) \subseteq \mathcal{X}_{\mathrm{f}}^{\text {glob }} \forall t \geq 0
$$

Proof: The proof is done by induction. By Definition 7, it holds that $\sum_{i=1}^{M} \alpha_{i}^{0} \leq \alpha$ and thus $\mathcal{X}_{\mathrm{f}, 1}\left(\alpha_{1}^{0}\right) \times \ldots \times$ $\mathcal{X}_{\mathrm{f}, M}\left(\alpha_{M}^{0}\right) \subseteq \mathcal{X}_{\mathrm{f}}^{\text {glob }}$. Assuming $\sum_{i=1}^{M} \alpha_{i}^{t} \leq \alpha$ at any time $t \geq 0$, due to (14) and (12d) it holds that

$$
\sum_{i=1}^{M} \alpha_{i}^{t+1}=\sum_{i=1}^{M} \alpha_{i}^{t}+\sum_{i=1}^{M} \gamma_{i}\left(x_{\mathcal{N}_{i}}^{t}\right) \leq \sum_{i=1}^{M} \alpha_{i}^{t} \leq \alpha
$$

which concludes the induction step and implies (17).

The results presented in Theorem 6, Lemma 8 and Lemma 9 provide the ingredients to prove recursive feasibility and asymptotic stability of the global closed-loop system $x^{t+1}=$ $A x^{t}+B \kappa_{\mathrm{MPC}}\left(x^{t}\right)$ in $\mathcal{X}_{N}$ using standard arguments (Mayne et al. 2000). Recursive feasibility follows from Lemma 8 and Lemma 9, asymptotic stability follows from Theorem 5 , as $\kappa_{\mathrm{f}}(x), V_{\mathrm{f}}(x)$ and $\mathcal{X}_{\mathrm{f}}^{\text {glob }}$ together fulfill (8).

\section{Distributed Synthesis of Cooperative Distributed MPC Controllers for Networks of Linear Systems}

In the previous section, a general formulation of a separable terminal cost and time-varying local terminal sets for the MPC problem (7) have been proposed, such that (7) is amenable to distributed optimization and stability and constraint satisfaction in closed-loop are guaranteed. In this section, a synthesis approach for the proposed terminal cost and set is presented, which consists of two parts. First, a distributed procedure to construct a separable quadratic Lyapunov function $V_{\mathrm{f}}(x)=x^{T} P_{\mathrm{f}} x$, i.e., with $P_{\mathrm{f}}$ block-diagonal, is presented in Section 4.1. Second, a distributed procedure 
to find $\mathcal{X}_{\mathrm{f}}^{\text {glob }}$ as the largest feasible level set of $V_{\mathrm{f}}(x)$ is presented in Section 4.2. Local terminal sets (13) follow from Definition 7 in a straightforward way.

Throughout this section, we consider linear systems, polytopic state and input constraints, as well as quadratic cost functions. In particular, the local constraints are of the form $\mathcal{X}_{i}=\left\{x_{i} \in \mathbb{R}^{n_{i}} \mid H_{x_{i}} x_{i} \leq h_{x_{i}}\right\}$ and $\mathcal{U}_{i}=\left\{u_{i} \in \mathbb{R}^{m_{i}} \mid H_{u_{i}} u_{i} \leq h_{u_{i}}\right\}$ for all $i \in \mathcal{M}$, where $H_{x_{i}} \in \mathbb{R}^{l_{x, i} \times n_{i}}, h_{x_{i}} \in \mathbb{R}^{l_{x, i}}, H_{u_{i}} \in \mathbb{R}^{l_{u, i} \times m_{i}}$ and $h_{u_{i}} \in \mathbb{R}^{l_{u, i}}$. Moreover, local stage cost functions are defined as $l_{i}\left(x_{\mathcal{N}_{i}}, u_{i}\right)=x_{\mathcal{N}_{i}}^{T} Q_{i} x_{\mathcal{N}_{i}}+u_{i}^{T} R_{i} u_{i}$, and local terminal cost functions are defined as $V_{\mathrm{f}, i}\left(x_{i}\right)=x_{i}^{T} P_{\mathrm{f}, i} x_{i}$ for all $i \in \mathcal{M}$. We define local relaxation functions $\gamma_{i}\left(x_{\mathcal{N}_{i}}\right)=x_{\mathcal{N}_{i}}^{T} \Gamma_{\mathcal{N}_{i}} x_{\mathcal{N}_{i}}$ for all $i \in \mathcal{M}$. Note that the matrices $Q_{i}, R_{i}$ and $P_{\mathrm{f}, i}$ are all positive definite, while $\Gamma_{\mathcal{N}_{i}}$ is allowed to be indefinite. Finally, we consider a structured linear terminal control law $\kappa_{\mathrm{f}}(x)$ according to Assumption 3.

In order to simplify the notation, we introduce lifting matrices $T_{i} \in\{0,1\}^{n_{i} \times n}, V_{i} \in\{0,1\}^{m_{i} \times m}$ and $W_{i} \in\{0,1\}^{n_{\mathcal{N}_{i}} \times n}$ for each $i \in \mathcal{M}$. These lifting matrices, similar to permutation matrices, have in each row exactly one entry equal to 1 , such that $x_{i}=T_{i} x, u_{i}=V_{i} u$ and $x_{\mathcal{N}_{i}}=W_{i} x$.

\subsection{Distributed Synthesis of Separable Terminal Cost}

In this section, a distributed synthesis method for a separable terminal cost is presented, which relies on the centralized synthesis tools introduced in Section 2.4.1. The main idea is to formulate a global synthesis problem such that it can be solved by distributed optimization without central coordination. In Section 4.1.1, a set of LMI conditions with one global coupling constraint is derived. In Section 4.1.2, a sufficient condition for this constraint is presented, exhibiting only neighbor-to-neighbor coupling.

\subsubsection{Structured LMIs with Global Coupling}

As discussed in Section 3.2, a sufficient condition for $V_{\mathrm{f}}(x)$ to be a Lyapunov function, is for the local cost terms to satisfy the conditions stated in (12). For the considered linear systems and quadratic functions, conditions (12c) and (12d) are equivalent to the matrix inequalities

$$
\begin{array}{r}
\left(A_{\mathcal{N}_{i}}+B_{i} K_{\mathcal{N}_{i}}\right)^{T} P_{\mathrm{f}, i}\left(A_{\mathcal{N}_{i}}+B_{i} K_{\mathcal{N}_{i}}\right)-\bar{P}_{\mathrm{f}, i} \leq \\
-\left(Q_{i}+K_{\mathcal{N}_{i}}^{T} R_{i} K_{\mathcal{N}_{i}}\right)+\Gamma_{\mathcal{N}_{i}}, \forall i \in \mathcal{M} \\
\sum_{i=1}^{M} W_{i}^{T} \Gamma_{\mathcal{N}_{i}} W_{i} \leq 0
\end{array}
$$

where $\bar{P}_{\mathrm{f}, i}:=W_{i} T_{i}^{T} P_{\mathrm{f}, i} T_{i} W_{i}^{T}$ is $P_{\mathrm{f}, i}$ lifted into the space of neighboring states. In the following, it will be demonstrated that conditions (18) can equivalently be written as a set of $M$ neighbor-to-neighbor coupled LMIs with one global constraint.
Lemma 10 Condition (18) is equivalent to the set of LMIs

$$
\left[\begin{array}{cccc}
\bar{E}_{i}+F_{\mathcal{N}_{i}} & E_{\mathcal{N}_{i}} A_{\mathcal{N}_{i}}^{T}+Y_{\mathcal{N}_{i}}^{T} B_{i}^{T} & E_{\mathcal{N}_{i}} Q_{i}^{1 / 2} & Y_{\mathcal{N}_{i}}^{T} R_{i}^{1 / 2} \\
A_{\mathcal{N}_{i}} E_{\mathcal{N}_{i}}+B_{i} Y_{\mathcal{N}_{i}} & E_{i} & 0 & 0 \\
Q_{i}^{1 / 2} E_{\mathcal{N}_{i}} & 0 & I_{\mathcal{N}_{i}} & 0 \\
R_{i}^{1 / 2} Y_{\mathcal{N}_{i}} & 0 & 0 & I_{m_{i}}
\end{array}\right]
$$

$\sum_{i=1}^{M} W_{i}^{T} F_{\mathcal{N}_{i}} W_{i} \leq 0$,

where $E_{i}:=P_{\mathrm{f}, i}^{-1}, \quad \bar{E}_{i}:=W_{i} T_{i}^{T} P_{\mathrm{f}, i}^{-1} T_{i} W_{i}^{T}, E_{\mathcal{N}_{i}}:=$ $W_{i} E W_{i}^{T}, F_{\mathcal{N}_{i}}:=E_{\mathcal{N}_{i}} \Gamma_{\mathcal{N}_{i}} E_{\mathcal{N}_{i}}$ and $Y_{\mathcal{N}_{i}}:=K_{\mathcal{N}_{i}} E_{\mathcal{N}_{i}}$ $\forall i \in \mathcal{M}$.

Proof: The equivalence (18a) $\Leftrightarrow$ (19a) is shown by left and right-multiplication of (18a) with $E_{\mathcal{N}_{i}}$ and applying the Schur complement twice. The equivalence (18b) $\Leftrightarrow$ (19b) follows from the fact that $\sum_{i=1}^{M} W_{i}^{T} \Gamma_{\mathcal{N}_{i}} W_{i} \Leftrightarrow$ $\sum_{i=1}^{M} W_{i}^{T} F_{\mathcal{N}_{i}} W_{i}$, highlighting the role of $F_{\mathcal{N}_{i}}$ as a substitution for $\Gamma_{\mathcal{N}_{i}}$ to achieve convexity in (19a). For details, see proof of Theorem IV.3 in (Conte et al. 2012).

Remark 11 The fact that distributed unconstrained controllers can be synthesized by distributed optimization methods has been recognized already in (Langbort et al. 2004). The synthesis problem presented here is however different in the sense that the increases in the local cost functions are explicitly bounded by quadratic functions $\gamma_{i}\left(x_{\mathcal{N}_{i}}\right)=$ $x_{\mathcal{N}_{i}}^{T} \Gamma_{\mathcal{N}_{i}} x_{\mathcal{N}_{i}}$, which are synthesized together with the terminal cost. These functions are important for the design of local time-varying terminal sets according to Definition 7.

Remark 12 Conditions (19) provide a constructive test for existence of a distributed unconstrained controller stabilizing the global system. Conservatism, compared to centralized linear controller synthesis, is introduced by (i) the choice of a block-diagonal quadratic Lyapunov function and (ii) the structured gain matrix $K_{\mathrm{f}}$. The derivation of general conditions for the existence of solutions to (19) is beyond the scope of this paper. Related literature includes (Šiljak 1991), where conditions on decentralized stabilizability are presented, and (Ho \& Chu 1972), where it is shown that for networks of partially nested systems, a distributed linear controller is optimal, provided the network is stabilizable.

In order to add a cost metric to the conditions in (19), we propose the maximization of the volume of the 1-level set of $V_{\mathrm{f}}(x)$. Maximizing this volume corresponds to minimizing the determinant of $P_{\mathrm{f}}$, which is equivalent to maximizing the determinant of $E=P_{\mathrm{f}}^{-1}$, which in turn can be achieved by maximizing the concave operator $\log \operatorname{det}(E)$. This operator, as stated in the following proposition, is separable for a block-diagonal $E$ and thus directly amenable to distributed optimization. 
Proposition 13 For a matrix $E=\operatorname{diag}_{i \in \mathcal{M}}\left(E_{i}\right)$, it holds that

$$
\log \operatorname{det}(E)=\sum_{i=1}^{M} \log \operatorname{det}\left(E_{i}\right)
$$

The synthesis of a structured Lyapunov function according to Theorem 6 can thus be posed as a decomposable convex optimization problem.

\subsubsection{Structured LMIs with Neighbor-to-Neighbor Cou- pling}

The main difficulty in the synthesis LMIs (19) is constraint (19b), which couples all systems in the network in one single constraint, as similarly recognized in (Hermans, Lazar \& Jokić 2010). In the following, we will describe a sufficient condition for (19b), which is based on neighbor-toneighbor coupling. Consider upper-bounding the matrices $F_{\mathcal{N}_{i}}$ by block-diagonal matrices $S_{\mathcal{N}_{i}}$, resulting in the conditions

$$
\begin{gathered}
F_{\mathcal{N}_{i}} \leq S_{\mathcal{N}_{i}} \forall i \in \mathcal{M}, \\
\sum_{i=1}^{M} W_{i}^{T} S_{\mathcal{N}_{i}} W_{i} \leq 0 \\
\Leftrightarrow \sum_{j \in \mathcal{N}_{i}} T_{j} W_{j}^{T} S_{\mathcal{N}_{j}} W_{j} T_{j}^{T} \leq 0 \quad \forall i \in \mathcal{M},
\end{gathered}
$$

where the equivalence in (21b) is due to $S_{\mathcal{N}_{i}}$ being blockdiagonal. The set of constraints (21) is clearly sufficient for (19b). Moreover, (21a) involves only local variables and the right hand side of (21b) represents $M$ LMIs, each of which involves variables of neighboring systems only, as exemplified in Fig. 1. Consequently, (21) provides sufficient conditions for replacing (19b), which renders the terminal cost synthesis problem amenable to distributed optimization under the given communication constraints.

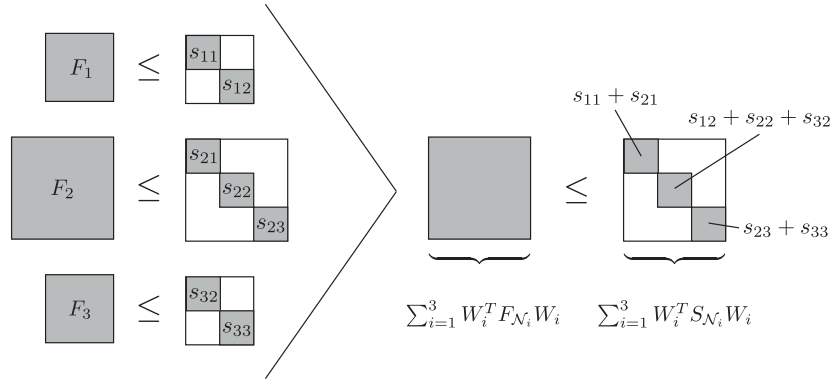

Fig. 1. Illustration of constraint (21) for a network of three systems with $\mathcal{N}_{1}=\{1,2\}, \mathcal{N}_{2}=\{1,2,3\}$ and $\mathcal{N}_{3}=\{2,3\}$.

\subsection{Distributed Synthesis of Structured Terminal Sets}

Given a separable terminal cost function $V_{\mathrm{f}}(x)=x^{T} P_{\mathrm{f}} x$ fulfilling condition (12), a feasible level set thereof can be used as a global set $\mathcal{X}_{\mathrm{f}}^{\text {glob }}=\left\{x \in \mathbb{R}^{n} \mid x^{T} P_{\mathrm{f}} x \leq \alpha\right\} \subseteq \mathcal{X}$, as required in Definition 7. Once such a global level set with size $\alpha$ is given, the initial local set sizes $\alpha_{i}^{0}$ can be allocated according to $\sum_{i=1}^{M} \alpha_{i}^{0} \leq \alpha$. The largest feasible level set of $V_{\mathrm{f}}(x)$ can be found, analogously to the centralized case described in Section 2.4.2, by solving the linear program

$$
\begin{aligned}
& \alpha_{\max }:=\max _{\alpha} \alpha \\
& \text { s.t. } \sigma_{\mathcal{E}}\left(H_{x_{i}, j}^{T}\right)^{2} \alpha=\left\|P_{\mathrm{f}, i}^{-\frac{1}{2}} H_{x_{i}, j}^{T}\right\|_{2}^{2} \alpha \leq h_{x_{i}, j}^{2} \\
& \forall i \in \mathcal{M}, \quad j \in\left\{1, \ldots, l_{x, i}\right\} \\
& \sigma_{\mathcal{E}}\left(K_{\mathcal{N}_{i}}^{T} H_{u_{i}, j}^{T}\right)^{2} \alpha=\left\|P_{\mathrm{f}, \mathcal{N}_{i}}^{-\frac{1}{2}} K_{\mathcal{N}_{i}}^{T} H_{u_{i}, j}^{T}\right\|_{2}^{2} \alpha \leq h_{u_{i}, j}^{2} \\
& \forall i \in \mathcal{M}, \quad j \in\left\{1, \ldots, l_{u, i}\right\},
\end{aligned}
$$

where $P_{\mathrm{f}, \mathcal{N}_{i}}:=W_{i} P_{\mathrm{f}} W_{i}^{T} \forall i \in \mathcal{M}$. The constraints (22b) and (22c) represent conditions on the support function of the $\alpha$-level set of $V_{\mathrm{f}}(x)$. In particular, (22b) ensures that any point $x \in \mathcal{X}_{\mathrm{f}}^{\text {glob }}$ fulfills the polytopic state constraints $x \in \mathcal{X}$ and (22c) ensures that the polytopic input constraints $K_{\mathrm{f}} x \in \mathcal{U}$ are satisfied. The structure in problem (22) allows for its solution by distributed optimization methods, e.g., as proposed in (Schizas, Ribeiro \& Giannakis 2008).

\section{Summary of Distributed Synthesis and Closed-Loop Operation of Nominal Cooperative Distributed MPC}

In this section, we briefly summarize the main steps of the proposed nominal cooperative distributed MPC for networks of linear systems. The procedure for distributed controller synthesis is described in Algorithm 1. In Step 1, a separable quadratic terminal cost $V_{\mathrm{f}}(x)=\sum_{i=1}^{M} x_{i}^{T} P_{i} x_{i}$, a structured linear terminal control law $\kappa_{\mathrm{f}}(x)=\operatorname{col}_{i \in \mathcal{M}}\left(K_{\mathcal{N}_{i}} x_{\mathcal{N}_{i}}\right)$ according to Assumption 3, and relaxation functions $\gamma_{i}\left(x_{\mathcal{N}_{i}}\right)=x_{\mathcal{N}_{i}}^{T} \Gamma_{\mathcal{N}_{i}} x_{\mathcal{N}_{i}} \forall i \in\{1, \ldots, M\}$ are computed, which satisfy condition (12). Subsequently, in Step 2, the maximum feasible level set of the global terminal cost function $V_{\mathrm{f}}(x)$ is found to serve as an outer bound $\mathcal{X}_{\mathrm{f}}^{\text {glob }}$ for the Cartesian product (15) of local terminal sets. Finally, a feasible initial configuration for the local terminal sets (13) is obtained in Step 3. The components constructed during the execution of Algorithm 1 can then directly be used in the MPC problem (7) to operate a stabilizing cooperative distributed MPC controller in closed-loop.

The main steps required for distributed closed-loop operation are summarized in Algorithm 2. Note that in Algorithm $2, u_{i}^{*}\left(x^{t}, 0\right)$ and $x_{\mathcal{N}_{i}}^{*}\left(x^{t}, N\right)$ are obtained from the optimal solution of the global MPC problem (7), which is solved by distributed optimization in Step 2, for the current state measurement $x^{t}$. In particular, given optimal finite-time input and state sequences $\mathbf{u}^{*}\left(x^{t}\right)=\left\{u^{*}\left(x^{t}, 0\right), \ldots, u^{*}\left(x^{t}, N-\right.\right.$ 1) $\}$ and $\mathbf{x}^{*}\left(x^{t}\right)=\left\{x^{*}\left(x^{t}, 0\right), \ldots, x^{*}\left(x^{t}, N\right)\right\}$, it holds that $u_{i}^{*}\left(x^{t}, 0\right)=V_{i} u^{*}\left(x^{t}, 0\right)$ and $x_{\mathcal{N}_{i}}^{*}\left(x^{t}, N\right)=W_{i} x^{*}\left(x^{t}, N\right)$. 
Algorithm 1 Offline synthesis of local terminal costs and time-varying local terminal sets as defined in Definition 7

1: Maximize (20) subject to (19a) and (21), by distributed optimization, to find $V_{\mathrm{f}, i}(x, i)=x_{i}^{T} P_{\mathrm{f}, i} x_{i}$ as well as $\kappa_{\mathcal{N}_{i}}\left(x_{\mathcal{N}_{i}}\right)$ and $\gamma_{i}\left(x_{\mathcal{N}_{i}}\right)=x_{\mathcal{N}_{i}}^{T} \Gamma_{\mathcal{N}_{i}} x_{\mathcal{N}_{i}}$ fulfilling (12), locally at each $i$ in $\{1, \ldots, M\}$.

2: Solve LP (22) by distributed optimization to find the largest feasible level set $\mathcal{X}_{\mathrm{f}}^{\text {glob }}=\left\{x \in \mathbb{R}^{n} \mid V_{\mathrm{f}}(x) \leq\right.$ $\left.\alpha_{\max }\right\}$, where $\alpha_{\max }$ is found locally at each system.

3: Find $\alpha_{i}^{0} \forall i \in\{1, \ldots, M\}$, such that $\sum_{i=1}^{M} \alpha_{i}^{0} \leq \alpha_{\max }$ (e.g. by setting $\alpha_{i}^{0}=\alpha / M$ for all $i$ ), to define initial local terminal sets (13).

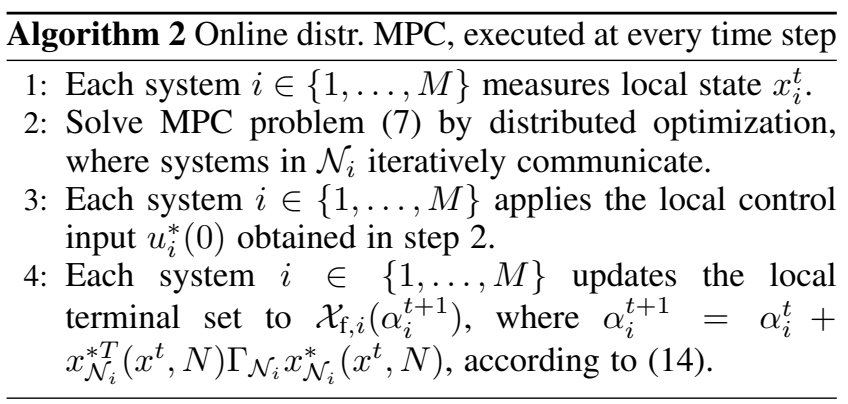

\section{Extension of Cooperative Distributed MPC to Refer- ence Tracking}

This section presents the main steps for extending the ideas presented in Section 3 and Section 4 to the problem of tracking piecewise constant references. The discussion covers common reference tracking formulations with focus on convergence guarantees. Offset-free techniques such as (Mäder, Borrelli \& Morari 2009) are not considered, but could be added to the proposed problem setup. In tracking MPC, the system output is required to follow a given reference $y^{t}$. We assume that for a given $y^{t}$, there exist points $\left(x_{\mathrm{s}}, u_{\mathrm{s}}\right)$ satisfying the global equilibrium condition

$$
\left[\begin{array}{cc}
A-I_{n} & B \\
C & 0
\end{array}\right]\left[\begin{array}{l}
x_{\mathrm{s}} \\
u_{\mathrm{s}}
\end{array}\right]=\left[\begin{array}{c}
0 \\
y^{t}
\end{array}\right] .
$$

Due to the structure in the matrices $A, B$ and $C$ defined in (1), (23) can be used, given a reference $y^{t}$ and using a separable objective function, to find an equilibrium pair $\left(x_{\mathrm{s}}, u_{\mathrm{s}}\right)$ by distributed optimization.

If an equilibrium pair $\left(x_{\mathrm{s}}, u_{\mathrm{s}}\right)$ is given, a distributed tracking MPC can be directly reformulated as distributed regulation MPC by defining the system state as $\Delta x:=x-x_{\mathrm{s}}$. For stability, as in the regulation case, a separable terminal cost and a structured terminal set are required. The main difficulty is the fact that the terminal set is centered around the target steady state $x_{\mathrm{s}}$. Thus, if $x_{\mathrm{s}}$ changes online, a new terminal set needs to be synthesized online, which may be prohibitively time-consuming. A solution to this problem, which will be further investigated in the following, is the use of a parameterized terminal set, as proposed in (Limon, Alvarado, Alamo \& Camacho 2008).

Definition 14 (Parametrized Invariant Set for Tracking) Let $\left(x_{s}, u_{s}\right)$ be an equilibrium point satisfying (23). Consider a control law $\kappa_{\mathrm{f}}(\cdot): \mathbb{R}^{n} \rightarrow \mathbb{R}^{m}$, which stabilizes the linear system $x^{+}=A x+B \kappa_{\mathrm{f}}(x)$. A set $\mathcal{X}_{\mathrm{tr}} \subseteq \mathbb{R}^{2 n+m}$ is a feasible invariant set for tracking, if the following conditions hold for each $\left(\Delta x, x_{s}, u_{s}\right) \in \mathcal{X}_{\mathrm{tr}}: \Delta x+x_{s} \in \mathcal{X}, u_{\mathrm{s}}+\kappa_{\mathrm{f}}(\Delta x) \in$ $\mathcal{U},\left(\Delta x, x_{s}, u_{s}\right) \in \mathcal{X}_{\mathrm{tr}} \Rightarrow\left(A \Delta x+B \kappa_{\mathrm{f}}(\Delta x), x_{s}, u_{s}\right) \in \mathcal{X}_{\mathrm{tr}}$.

\subsection{Formulation of Parametrized Local Time-Varying Ter- minal Sets for Cooperative Distributed Tracking MPC}

A separable terminal cost $V_{\mathrm{f}}(\Delta x)$ satisfying (12) under the assumptions of Theorem 6 can be defined and computed as proposed in Section 3 and Section 4. A parametrized set according to Definition 14 can then be defined as a level set of the function

$$
\begin{array}{r}
V_{\mathrm{tr}}\left(\Delta x, x_{\mathrm{s}}, u_{\mathrm{s}}\right):=V_{\mathrm{f}}(\Delta x)+V_{x}\left(x_{\mathrm{s}}\right)+V_{u}\left(u_{\mathrm{s}}\right) \\
=\Delta x^{T} P_{\mathrm{f}} \Delta x+x_{\mathrm{s}}^{T} P_{x} x_{\mathrm{s}}+u_{\mathrm{s}}^{T} P_{u} u_{\mathrm{s}}
\end{array}
$$

where $P_{\mathrm{f}}:=\operatorname{diag}_{i \in \mathcal{M}}\left(P_{\mathrm{f}, i}\right), P_{x}:=\operatorname{diag}_{i \in \mathcal{M}}\left(P_{x, i}\right), P_{u}:=$ $\operatorname{diag}_{i \in \mathcal{M}}\left(P_{u, i}\right)$, and where $P_{\mathrm{f}, i}, P_{x, i} \subseteq \mathbb{R}^{n_{i}}$ and $P_{u, i} \subseteq \mathbb{R}^{m_{i}}$ $\forall i \in \mathcal{M}$. Note that under the assumptions of Theorem 6 and due to the properties (12) of $V_{\mathrm{f}}(\Delta x)$, any feasible level set $\mathcal{X}_{\mathrm{tr}}^{\text {glob }}:=\left\{\left(\Delta x, x_{\mathrm{s}}, u_{\mathrm{s}}\right) \in \mathbb{R}^{2 n+m} \mid V_{\mathrm{tr}}\left(\Delta x, x_{\mathrm{s}}, u_{\mathrm{s}}\right) \leq \alpha\right\}$ is invariant in the sense of Definition 14, independent of how $V_{x}\left(x_{\mathrm{s}}\right)$ and $V_{u}\left(u_{\mathrm{s}}\right)$ are chosen.

Due to the block-diagonal property of $P_{\mathrm{f}}, P_{x}$ and $P_{u}$, local time-varying terminal sets for tracking, which are locally parametrized in $\left(x_{\mathrm{s}, i}, u_{\mathrm{s}, i}\right) \forall i \in \mathcal{M}$, can be defined as

$$
\begin{aligned}
& \mathcal{X}_{\mathrm{tr}, i}\left(\alpha_{i}^{t}\right)=\left\{\left(\Delta x_{i}, x_{\mathrm{s}, i}, u_{\mathrm{s}, i}\right) \in \mathbb{R}^{2 n_{i}+m_{i}}\right. \\
& \left.\Delta x_{i}^{T} P_{\mathrm{f}, i} \Delta x_{i}+x_{\mathrm{s}, i}^{T} P_{x, i} x_{\mathrm{s}, i}+u_{\mathrm{s}, i}^{T} P_{u, i} u_{\mathrm{s}, i} \leq \alpha_{i}^{t}\right\} \forall i \in \mathcal{M}
\end{aligned}
$$

where $\alpha_{i}^{t}$ is time-varying according to (14). Similarly as in the regulation case in Lemma 8 , it can be shown that if the point $\left(\Delta x_{i}, x_{\mathrm{s}, i}, u_{\mathrm{s}, i}\right)$ is contained in $\mathcal{X}_{\mathrm{tr}, i}\left(\alpha_{i}^{t}\right)$, then the point $\left(A_{\mathcal{N}_{i}} \Delta x+B_{i} \kappa_{\mathcal{N}_{i}}\left(\Delta x_{\mathcal{N}_{i}}\right), x_{\mathrm{s}, i}, u_{\mathrm{s}, i}\right)$ will be contained in $\mathcal{X}_{\mathrm{tr}, i}\left(\alpha_{i}^{t+1}\right)$. Furthermore, as in the regulation case in Lemma 9, given a feasible global invariant set for tracking $\mathcal{X}_{\mathrm{tr}}^{\text {glob }}$, it can be shown that if $\alpha_{1}^{0}+\ldots+\alpha_{M}^{0} \leq \alpha$, then the Cartesian product $\mathcal{X}_{\text {tr }, 1}\left(\alpha_{1}^{t}\right) \times \ldots \times \mathcal{X}_{\text {tr }, M}\left(\alpha_{M}^{t}\right)$ of local time-varying terminal sets will remain within $\mathcal{X}_{\mathrm{tr}}^{\text {glob }}$ at all times $t \geq 0$. Thus, the main nominal results from Section 3 extend to the reference tracking case, see also (Conte, Zeilinger, Morari \& Jones 2013) for more details.

\section{Numerical Examples}

In this section, the methodology for cooperative distributed MPC presented in this paper is illustrated by a numerical 
example simulating the behavior of a chain of masses $m=$ $1 \mathrm{~kg}$, connected by springs with $k=3 \mathrm{~N} / \mathrm{m}$ and dampers with $b=3 \mathrm{Ns} / \mathrm{m}$, see Fig. 2. The continuous-time ODEs describing the dynamics are discretized by the forward Euler method with a sampling time of $0.2 \mathrm{~s}$. The resulting linear discrete-time system is of form (1), with $x_{i}$ containing the position and velocity of mass $i$ and $u_{i}$ being a force applied to mass $i$. Constraints are imposed as $\left\|x_{i}\right\|_{\infty} \leq 10$ and $\left\|u_{i}\right\|_{\infty} \leq 1, Q_{\mathcal{N}_{i}}$ and $R_{i}$ are identity, and the MPC horizon is $N=5$. The local terminal cost terms $V_{\mathrm{f}, i}\left(x_{i}\right)$ as well as the local relaxation functions $\gamma_{i}\left(x_{\mathcal{N}_{i}}\right)$ are designed to be quadratic functions as introduced in Section 4.

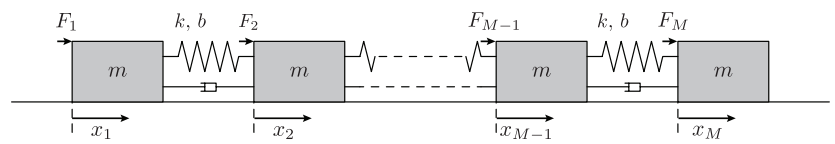

Fig. 2. Chain of masses, connected by spring and damper elements.

The closed-loop behavior for basic regulation to the origin is investigated on a chain of $M=5$ masses, comparing three controller setups: (i) centralized MPC with a terminal set defined by the maximum volume feasible ellipsoid characterized by a dense Lyapunov matrix $P$, (ii) distributed MPC as proposed in this paper and (iii) distributed MPC with the origin as a trivial terminal set. The initial states are zero for all systems except for the first one, i.e., $x_{1}=[0.27 \mathrm{~m}, 0 \mathrm{~m} / \mathrm{s}]^{T}$. The system is simulated for 15 steps, its closed-loop behavior is illustrated in Fig. 3. Note that the trajectories for setups (i) and (ii) are quite similar, illustrating the proximity in performance between (i) and (ii). Furthermore, Fig. 3(c) depicts the terminal set sizes of masses 1,2 and 5 under distributed MPC as proposed in this paper. Note that the local terminal set sizes change considerably during convergence of the closed-loop system.

The region of attraction (RoA) of the closed-loop system is analyzed in Fig. 4, showing that for short prediction horizons, there is a significant advantage in terms of RoA size when non-trivial, locally time-varying terminal sets are used instead of single-point terminal sets. For long prediction horizons, the RoAs of both controllers converge to the maximum RoA for the given constrained system. Note that each data point in Fig. 4 is a relative estimate of the extension of the RoA, either for controller (ii) or (iii) under a given horizon length $N$. Each estimate is obtained as the average of RoA extensions in 100 random directions. The estimate is relative as the averages for setup (ii) and (iii) is normalized by the respective average for controller setup (i).

\section{Conclusions}

In this paper, two contributions regarding stability and synthesis of cooperative distributed MPC were presented: (i) the formulation of a cooperative distributed MPC controller, which is stabilizing and suitable for distributed optimization in closed-loop and (ii) the synthesis procedure for such a controller, which can be executed in a purely distributed

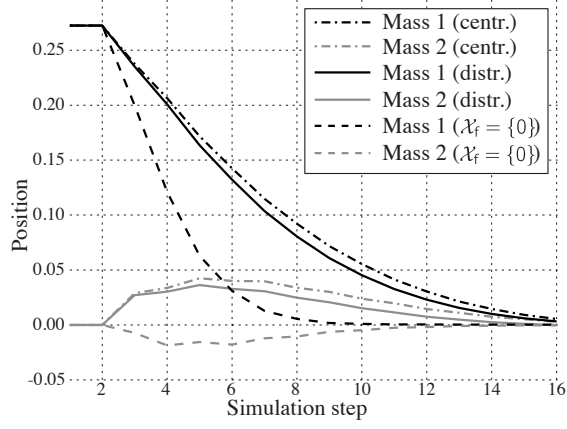

(a) Mass position trajectories.

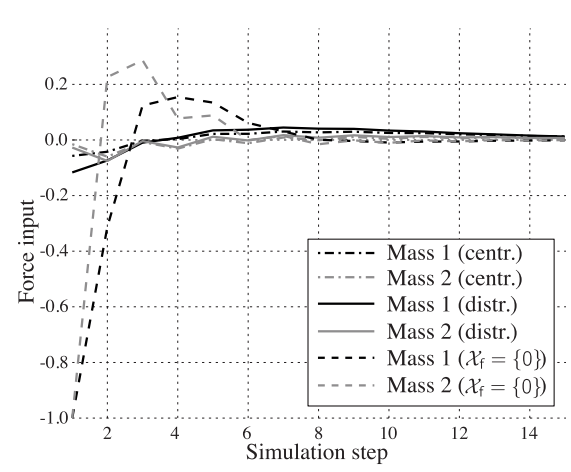

(b) Input trajectories.

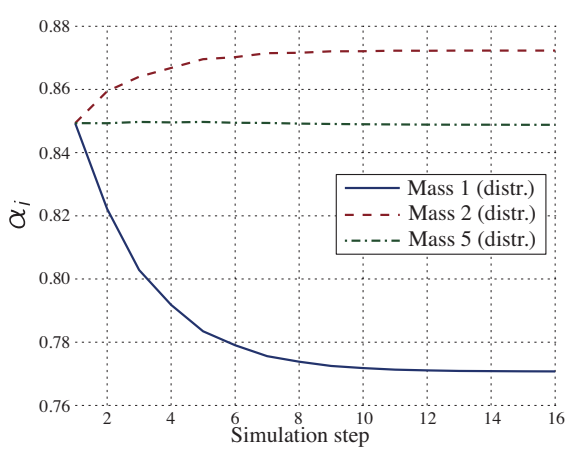

(c) Local terminal set sizes.

Fig. 3. Trajectories under nominal cooperative distributed MPC for regulation.

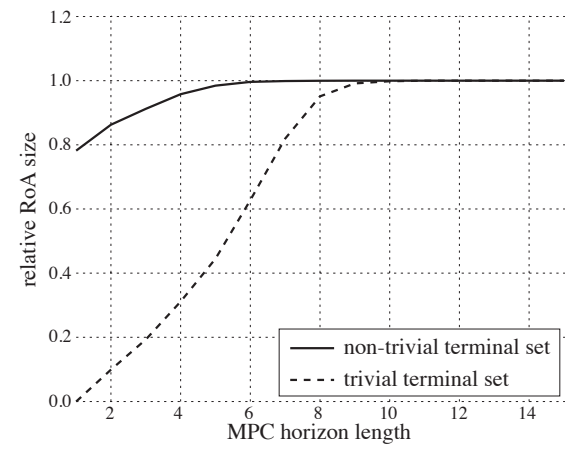

Fig. 4. Estimates of the relative RoA size for cooperative distributed MPC with non-trivial and trivial terminal conditions. 
manner. The combination of (i) and (ii) yields a framework for cooperative distributed MPC without central coordination, both for a nominal and for a reference tracking setup.

\section{Acknowledgement}

The work of C.N. Jones has received funding from the European Research Council under the European Unions Seventh Framework Programme (FP/2007-2013)/ ERC Grant Agreement n. 307608.

\section{References}

Bertsekas, D. P. \& Tsitsiklis, J. N. (1989), Parallel and Distributed Computation, Prentice Hall Inc., Old Tappan, NJ (USA).

Blanchini, F. (1999), 'Set invariance in control', Automatica 35, 1747 1767.

Boyd, S., Ghaoui, L. E., Feron, E. \& Balakrishnan, V. (1994), Linear Matrix Inequalities in System and Control Theory, Vol. 15 of Studies in Applied Mathematics, Society for Industrial and Applied Mathematics (SIAM).

Conte, C., Voellmy, N. R., Zeilinger, M. N., Morari, M. \& Jones, C. N. (2012), Distributed synthesis and control of constrained linear systems, in 'Proceedings of the American Control Conference', Montreal, Canada, pp. $6017-6022$.

Conte, C., Zeilinger, M. N., Morari, M. \& Jones, C. N. (2013), Cooperative distributed tracking MPC for constrained linear systems: Theory and synthesis, in 'Proceedings of the 52nd Conference on Decision and Control', Florence, Italy, pp. 3812 - 3817.

Farina, M. \& Scattolini, R. (2012), 'Distributed predictive control: A noncooperative algorithm with neighbor-to-neighbor communication for linear systems', Automatica 48(6), 1088 - 1096.

Giselsson, P. \& Rantzer, A. (2013), 'On feasibility, stability and performance in distributed model predictive control', IEEE Transactions on Automatic Control 59(4), 1031 - 1036.

Hermans, R. M., Lazar, M. \& Jokić, A. (2010), Almost decentralized Lyapunov-based nonlinear model predictive control, in 'Proceedings of the American Control Conference', Baltimore, Maryland, pp. $3932-3938$.

Ho, Y. \& Chu, K. (1972), 'Team decision theory and information structures in optimal control problems - part I', IEEE Transactions on Automatic Control 17(1), 15 - 22.

Jokić, A. \& Lazar, M. (2009), On decentralized stabilization of discretetime nonlinear systems, in 'Proceedings of the American Control Conference', St. Louis, MO, USA, pp. 5777 - 5782.

Langbort, C., Chandra, R. S. \& D'Andrea, R. (2004), 'Distributed control design for systems interconnected over an arbitrary graph', IEEE Transactions on Automatic Control 49(9), 1502 - 1519.

Limon, D., Alvarado, I., Alamo, T. \& Camacho, E. (2008), 'MPC for tracking piecewise constant references for constrained linear systems', Automatica 44(9), 2382 - 2387.

Ma, Y., Richter, S. \& Borrelli, F. (2012), 'Distributed model predictive control for building temperature regulation', SIAM Control and Optimization with Differential-Algebraic Constraints 23, 293 - 314.

Mäder, U., Borrelli, F. \& Morari, M. (2009), 'Linear offset-free model predictive control', Automatica 45(10), 2214 - 2222.

Maestre, J. M., Muñoz de la Peña, D., Camacho, E. F. \& Alamo, T. (2011), 'Distributed model predictive control based on agent negotiation', Journal of Process Control 21, 685 - 697.
Mayne, D. Q., Rawlings, J. B., Rao, C. V. \& Scokaert, P. O. M. (2000), 'Constrained model predictive control: Stability and optimality', Automatica 36, 789 - 814.

Raković, S. V., Kern, B. \& Findeisen, R. (2010), Practical set invariance for decentralized discrete time systems, in 'Proceedings of the 49th IEEE Conference on Decisions and Control', Atlanta, GA, USA, pp. $3283-3288$.

Riverso, S., Farina, M. \& Trecate, G. F. (2013), 'Plug-and-play decentralized model predictive control for linear systems', IEEE Transactions on Automatic Control 58(10), 2608 - 2614.

Schizas, I. D., Ribeiro, A. \& Giannakis, G. B. (2008), 'Consensus in ad hoc WSNs with noisy links - part I: Distributed estimation of deterministic signals', IEEE Transactions on Signal Processing 56(1), 350 - 364.

Stewart, B. T., Venkat, A. N., Rawlings, J. B., Wright, S. J. \& Pannocchia, G. (2010), 'Cooperative distributed model predictive control', Systems \& Control Letters 59, 460 - 469.

Venkat, A. N., Hiskens, I. A., Rawlings, J. B. \& Wright, S. J. (2008), 'Distributed MPC strategies with application to power system automatic generation control', IEEE Transactions on Control Systems Technology 16(6), 1192 - 1206.

Venkat, A. N., Rawlings, J. B. \& Wright, S. J. (2005), Stability and optimality of distributed model predictive control, in 'Proceedings of the 44th IEEE Conference on Decision and Control', Seville, Spain, pp. $6680-6685$.

Šiljak, D. D. (1991), Decentralized Control of Complex Systems, Vol. 184 of Mathematics in Science and Engineering, Academic Press, Inc., Boston.

Zeilinger, M., Pu, Y., Riverso, S., Ferrari-Trecate, G. \& Jones, C. N. (2013), Plug and play distributed model predictive control based on distributed invariance and optimization, in 'Proceedings of the 52nd Conference on Decision and Control', Florence, Italy, pp. 5770 5776.

Zečević, A. I. \& Šiljak, D. D. (2010), Control of Complex Systems, Communications and Control Engineering, Springer, New York. 